\title{
Ejection-Collision orbits in the symmetric collinear four-body problem
}

\author{
M. Alvarez-Ramírez \\ Dept. de Matemáticas, UAM-Iztapalapa, 09340 Iztapalapa, Ciudad de México, México \\ E. Barrabés \\ Dept. Informàtica Matemàtica Aplicada i Estadística, Universitat de Girona, Girona, Spain \\ M. Medina \\ Dept. de Matemáticas, UAM-Iztapalapa, 09340 Iztapalapa, Ciudad de México, México \\ M. Ollé \\ Dept. Matemàtiques Universitat Politècnica de Catalunya, \\ Av Diagonal 647, Barcelona 08028, Spain
}

\begin{abstract}
In this paper, we consider the collinear symmetric four-body problem, where four masses $m_{3}=\alpha, m_{1}=1, m_{2}=1$, and $m_{4}=\alpha, \alpha>0$, are aligned in this order and move symmetrically about their center of mass. We introduce regularized variables to deal with binary collisions as well as McGehee coordinates to study the quadruple collision manifold for a negative value of the energy. The paper is mainly focused on orbits that eject from (or collide to) quadruple collision. The problem has two hyperbolic equilibrium points, located in the quadruple collision manifold. We use high order parametrizations of their stable/unstable manifolds to devise a numerical procedure to compute ejectioncollision orbits, for any value of $\alpha$. Some results from the explorations done for $\alpha=1$ are presented. Furthermore, we prove the existence of ejection-direct escape orbits, which perform a unique type of binary collisions.
\end{abstract}

Keywords: collinear four-body problem, ejection/collision orbits, binary

Email addresses: mar@xanum.uam.mx (M. Alvarez-Ramírez), esther.barrabes@udg.edu (E. Barrabés), mvmg@xanum.uam.mx (M. Medina), Merce.0lle@upc.edu (M. Ollé)

Preprint submitted to Journal of ${ }^{A} T_{E} X$ Templates

November 12, 2018 
collisions, invariant manifolds, escape criteria

2010 MSC: 00-01, 99-00

\section{Introduction}

The classical $n$-body problem studies the dynamics of $n$ point masses interacting according to Newtonian gravity. In the symmetric collinear four-body problem, the bodies are symmetrically distributed about the centre of mass by 5 pairs, each of those pairs have equal mass and the configuration of the four bodies is collinear at every instant. It is a two degrees of freedom problem which is a sub-problem of the trapezoidal four-body problem that has three degrees of freedom, see the works of Lacomba and Simó ([1, 2]).

The four-body problem has attracted the attention of numerous astronomers since through it, the gravitational interaction of many stellar or exoplanetary systems can be modelled, as the interaction of two binary star systems or the interplay of two planets with a binary star system. Many of the studies, as the influence between two binaries, have been carried out from the numerical point of view ( [3], [4] , [5, [6]). Also, the close interaction of systems of few stars give

15 rise to the possibility of collisions between two or more stars in a cluster, as close encounters and direct physical collisions between stars are frequent in globular clusters, [7. These collisions are more frequent as a binary-binary system than as a system formed by a single star and a binary one. Other numerical studies have been conducted to understand the numerical scattering of the influence between binary-binary or single-binary systems, see [8].

We focus on the particular case of the collinear model of a four body problem. A solution of the symmetric collinear four-body problem, denoted by SC4BP, experiences a collision if two or more particles come together at a certain time. At such a time the potential energy approaches infinity, the equations of motion become undefined and the solution has a singularity. The analytical and numerical study of this problem requires the McGehee's blow up technique to regularize the singularity corresponding to total (quadruple) collision and the 
regularization of binary collisions (collisions between $m_{1}$ and $m_{2}$ ) and simultaneous binary collisions $\left(m_{1}\right.$ and $m_{3}$ collide as well as $m_{2}$ and $\left.m_{4}\right)$, see for example [9] and [10]. This singularity due to total collision is blown up and in its place is glued an invariant total collision manifold. In [2], Simó and Lacomba analyze the flow on the total collision manifold and they find a family of connection orbits between two quadruple collisions which arise as the parameter of masses is varied. The flow on this manifold provides relevant information for the flow close to quadruple collision.

In addition to the mentioned works of Simó and Lacomba, several papers can be found in the literature on the symmetric collinear four-body problem. Sweatman [1] studies the symmetrical collinear four-body problem with equal masses. He finds very interesting dynamical phenomena for the problem under study, 40 showing the existence of periodic, quasiperiodic, fast-scattering and chaoticscattering orbits. Still in the case of equal masses, Sekiguchi and Tanikawa [12] study the SC4BP both analytically and numerically. In particular, they classify a great variety of orbits by means of symbol sequences and they obtain the initial conditions leading to escape using escape criteria established in the 45 paper.

Focussing on periodic orbits, Bakker et al. [13] and Sweatman [14, using analytic-numerical methods, study the existence of Schubart-like orbits (that is, periodic solutions with exactly two binary collisions and one simultaneous binary collision per period) as well as their stability depending on the mass parameter. Later on, Ouyang and Yan [15] and Huang [16] analytically prove the existence of such Schubart periodic orbits by applying topological methods and variational calculus, respectively.

We finally mention, for the symmetric collinear four-body problem, the papers by Alvarez et al. [9, 17, where the authors provide some analytical results concerning singularities and regularization, and analytically study the quadruple collision manifold, the equilibrium points, the infinity manifold and the relation between both manifolds which allow them to prove the existence of orbits connecting quadruple collision and infinity. For the collinear non sym- 
metric four-body problem, in [18, Mather and McGehee prove the existence of solutions which become unbounded in finite time for special values of the masses.

The main goal in this paper is to amalgamate both theoretical and numerical tools to investigate, on one hand, orbits that eject from quadruple collision and have a fast escape to infinity, and, on the other hand, ejection-collision orbits (also denoted by ECO), that is orbits that eject from quadruple collisions and go back to quadruple collision. The latter are regarded as heteroclinic connections between the two equilibrium points (that lie on the total collision manifold). In order to numerically compute ejection-escape orbits and ejection-collision ones we need the construction of parametrizations, up to certain order, of the 70 stable and unstable invariant manifolds, $W^{s}$ and $W^{u}$, of the equilibrium points, using the methodology explained in [19]. At this point we mention the work by Sekiguchi and Tanikawa 12 where due to the Poincaré section considered, the ejection-collision orbits are all mixed up and indistinguishable. In the present paper a different Poincaré section has been taken into account that allows to classify and distinguish different types of ejection-collision orbits. Following this classification, In 20] Lacomba and Medina proved analytically the existence of certain ejection collision orbits for specific values of the mass parameter. In this paper, a numerical method is explained to compute ECO for any value of the mass parameter $\alpha$ and negative energy $h$. The results are presented for $\alpha=1$ so and $h=-1$.

The paper is organized as follows: in Section 2 we recall briefly some known results about the dynamics of the SC4BP, including the regularization of total collision using McGehee's coordinates [10, the regularization of binary collisions, the description of the flow on the quadruple collision manifold and 85 the existence of two hyperbolic equilibrium points, $E^{ \pm}$. We compute high order parametrizations of the associated stable and unstable invariant manifolds, $W^{s, u}\left(E^{ \pm}\right)$and some error tests have been carried out to control the accuracy of the approximations. Section 3 is devoted to the orbits that eject from (or collide to) quadruple collision and directly escape to (come from) infinity describing a 
unique type of binary collisions. In Section 4 we present some properties of the ejection-collision orbits and devise a numerical method to compute them. We show the results for the case $\alpha=1$.

\section{The symmetric collinear four body-problem}

The aim of this section is to present a summary of the equations and the main properties of the symmetric collinear four-body problem, SC4BP. For more details see, for example, [17, 2] and the references therein. In particular, we focus on the main features for the computation of ejection/collision orbits (orbits that start/end at a quadruple collision) and ejection-collision orbits (ECO, orbits that start and end at a quadruple collision): the dynamics on the total collision manifold, the stable and unstable invariant manifolds of the equilibrium points and the Poincaré section used.

\subsection{Equations of the $S C 4 B P$}

The symmetric collinear four-body problem consists of four point masses, with masses $m_{i}, i=1, \ldots, 4$, moving on a straight line under the Newton's law of gravitation with $m_{1}=m_{2}$ and $m_{3}=m_{4}$ in symmetric positions with respect to the center of mass at the origin. Without loss of generality, we can suppose that the first two bodies have mass $m_{1}=m_{2}=1$ and are located at $\pm x$, and the other two bodies have mass $m_{3}=m_{4}=\alpha$, where $\alpha \in(0, \infty)$, and are located at $\pm y / \sqrt{\alpha}$. See Figure 1 .

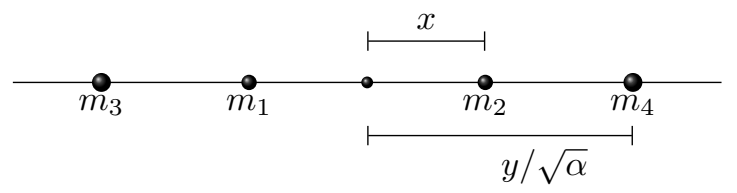

Figure 1: Symmetric collinear four-body problem.

In this set of coordinates, the Hamiltonian of the problem is given by

$$
H\left(x, y, p_{x}, p_{y}\right)=\frac{p_{x}^{2}}{4}+\frac{p_{y}^{2}}{4}-U(x, y),
$$


where $p_{x}=2 \frac{d x}{d t}, p_{y}=2 \frac{d y}{d t}$ and the potential function is

$$
U(x, y)=\frac{1}{2 x}+\frac{\alpha^{5 / 2}}{2 y}+\frac{2 \alpha^{3 / 2}}{y-\sqrt{\alpha} x}+\frac{2 \alpha^{3 / 2}}{y+\sqrt{\alpha} x} .
$$

110

The phase space of the problem is $\mathcal{U} \times \mathbb{R}^{2}$ where $\mathcal{U}=\left\{(x, y) \in \mathbb{R}^{2} \mid 0<\sqrt{\alpha} x<y\right\}$.

Notice that the equations have three singularities, one at $x=0$ and $y \neq 0$, another at $y=\sqrt{\alpha} x \neq 0$, and a third one corresponding to $x=y=0$. They correspond to the following collision configurations:

- Single binary collision: the bodies $m_{1}$ and $m_{2}$ collide, while the other two bodies remain bounded away from them. This type of collision corresponds to $x=0$ and $y \neq 0$ (collision of type 1 or SBC).

- Double (simultaneous) binary collision: the bodies $m_{1}$ and $m_{3}$ collide, and by the symmetry of the problem, so do the other two bodies. This double collision corresponds to $y=\sqrt{\alpha} x \neq 0$ (collision of type 2 or $\mathrm{DBC})$.

- Quadruple collision: the four bodies collide. This collision corresponds to $x=y=0$.

We fix a value of the energy in $H=h$, so the motion takes place in a 3 dimensional manifold and, using (1), it is confined in the configuration space $(x, y)$ to the Hill's region given by

$$
\mathcal{R}_{h}=\{(x, y) \in \mathcal{U} \mid U(x, y) \geq-h\} .
$$

The function $U(x, y)$ (given in (2)) is strictly positive for all $(x, y) \in \mathcal{U}$, so for $h \geq 0$ the Hill's region coincides with the configuration space $\mathcal{U}$, whereas for $h<0$ the Hill's region is limited by $U(x, y)=-h$. In Figure 2 we show the Hill's region for a negative value of the energy $h$, and an orbit which ends at the quadruple collision and performs different binary collisions. We will use the representation of the orbits in the configuration space $(x, y)$ inside the Hill's region $\mathcal{R}_{h}$ through the paper. 


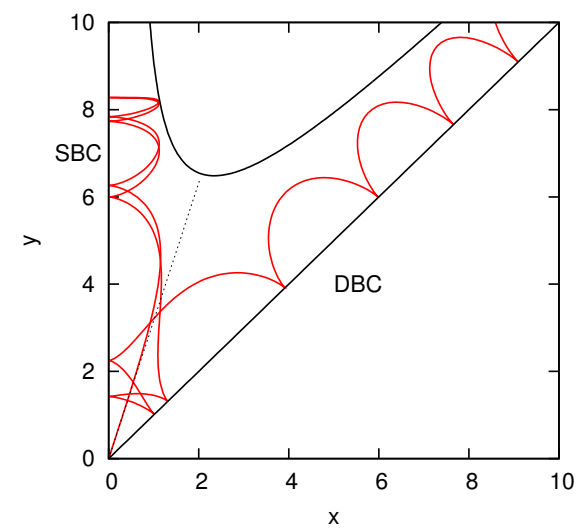

Figure 2: Zero velocity curve and Hill's region (defined in (3) in the configuration space $(x, y)$, the homothetic solution (with $\theta=\theta_{c}$, dotted line, see Section 2.2 , and a solution of the SC4BP with single binary collisions (SBC, at $x=0)$ and double binary collisions (DBC, at $y=\sqrt{\alpha} x)$ for $\alpha=1$ and $h=-1$.

In the $N$-body problem bounded motions can only occur if $h<0$ (see, for example Chapter 4 in 21]). Therefore, to study the ejection-collision orbits we consider only negative values of the energy.

Furthermore, in order to study the dynamics close to the quadruple collision, it is necessary to describe the total collision manifold and the flow on it. 135 For this purpose, we apply the blow-up technique introduced by McGehee [10]. Moreover, since the solutions of the SC4BP typically perform several binary collisions, we will also regularize the singularities (due to collisions) that appear in the system of the ordinary of differential equations (ODE). The suitable transformations of the blow up and the regularization in the symmetric collinear four-body problem have been made by Alvarez-Ramírez et al. [17. For completeness of the present work, and in order to understand the meaning of the regularized variables, we summarize the changes carried out.

- Introduce polar coordinates

$$
\begin{array}{ll}
x=\frac{r}{\sqrt{2}} \cos \theta, & y=\frac{r}{\sqrt{2}} \sin \theta, \\
p_{x}=\sqrt{2} p_{r} \cos \theta-\sqrt{2} p_{\theta} \sin \theta, & p_{y}=\sqrt{2} p_{r} \sin \theta+\frac{\sqrt{2}}{r} p_{\theta} \cos \theta,
\end{array}
$$


$\theta \in\left(\theta_{\alpha}, \frac{\pi}{2}\right)$, where $\theta_{\alpha}=\arctan (\sqrt{\alpha})$ corresponds to double binary collisions (DBC). The associated potential function is

$$
V(\theta)=r U(x, y)=\frac{1}{\sqrt{2} \cos \theta}+\frac{\alpha^{5 / 2}}{\sqrt{2} \sin \theta}+\frac{2 \sqrt{2} \alpha^{3 / 2}}{\sin \theta-\sqrt{\alpha} \cos \theta}+\frac{2 \sqrt{2} \alpha^{3 / 2}}{\sin \theta+\sqrt{\alpha} \cos \theta} .
$$

- Introduce the McGehee's coordinates $(v, u)$ and a change in time through the relations

$$
p_{r}=r^{-1 / 2} v, \quad p_{\theta}=r^{1 / 2} u, \quad d t=r^{3 / 2} d \tau .
$$

- Remove simultaneously all binary collisions, considering the regularized potential

$$
W(\theta)=V(\theta) \cos \theta(\sin \theta-\sqrt{\alpha} \cos \theta)
$$

which is a positive, real analytic function in $\left[\theta_{\alpha}, \pi / 2\right]$, and the change of time and coordinates given by

$$
\frac{d \tau}{d s}=\Delta(\theta)=\frac{\cos \theta(\sin \theta-\sqrt{\alpha} \cos \theta)}{\sqrt{W(\theta)}}, \quad w=\Delta(\theta) u
$$

In coordinates $(r, v, \theta, w)$ and $s$ as time variable, the equations of the SC4BP become

$$
\begin{aligned}
\frac{d r}{d s}= & r v \Delta(\theta), \\
\frac{d v}{d s}= & \sqrt{W(\theta)}+\left(2 r h-\frac{v^{2}}{2}\right) \Delta(\theta), \\
\frac{d \theta}{d s}= & w, \\
\frac{d w}{d s}= & -\frac{v w}{2} \Delta(\theta)+(\cos 2 \theta+\sqrt{\alpha} \sin 2 \theta)\left(\frac{2 r h-v^{2}}{\sqrt{W(\theta)}} \Delta(\theta)+1\right) \\
& \quad+\frac{W^{\prime}(\theta)}{W(\theta)}\left(\cos \theta(\sin \theta-\sqrt{\alpha} \cos \theta)-\frac{w^{2}}{2}\right)
\end{aligned}
$$

where $W^{\prime}=\frac{d W}{d \theta}$. The energy relation $H=h$ from 11 becomes

$$
w^{2}=\left(2 r h-v^{2}\right) \Delta(\theta)^{2}+2 \cos \theta(\sin \theta-\sqrt{\alpha} \cos \theta) .
$$


The vector field defined by equations $(6)$ is an analytic vector field on the phase space $\mathcal{F}=[0, \infty) \times \mathbb{R} \times\left[\theta_{\alpha}, \pi / 2\right] \times \mathbb{R}$. The solutions of the ODE, also called orbits, will be denoted by $\Gamma=\{\gamma(s)\}_{s \in \mathbb{R}}$ or simply by $\gamma(s)$.

A straightforward computation shows that the set of equations (6) satisfies the symmetry

$$
\mathcal{L}_{1}: \quad(r, v, \theta, w, s) \rightarrow(r,-v, \theta,-w,-s) .
$$

Therefore, if $\Gamma$ is a solution given by $\gamma(s)=(r(s), v(s), \theta(s), w(s))$, then $\bar{\Gamma}$ defined as

$$
\bar{\gamma}(s)=(r(-s),-v(-s), \theta(-s),-w(-s))
$$

\subsection{Total collision manifold, equilibrium points and invariant manifolds}

Notice that the system (6) is well defined for $r=0$, which corresponds to the total collision manifold $\mathcal{C}$, given by

$$
\mathcal{C}=\left\{(r, v, \theta, w) ; r=0, \quad w^{2}=-\Delta(\theta)^{2} v^{2}+2 \cos \theta(\sin \theta-\sqrt{\alpha} \cos \theta)\right\},
$$

which is a 2-dimensional manifold, topologically equivalent to a sphere minus four points, independent of the total energy $h$ and invariant under the flow (6). The total collision manifold $\mathcal{C}$ belongs to the boundary of the manifold 
$\mathcal{C}$ is gradient-like with respect the variable $v$, that is, $d v / d s \geq 0$. See, for more details, [17, 22, 2].

The SC4BP has two equilibrium points

$$
E^{ \pm}=\left(0, \pm v_{c}, \theta_{c}, 0\right)
$$

where $\theta_{c}$ is the only solution of $V^{\prime}(\theta)=0$ (the potential $V$ is defined in (4)). Furthermore, the SC4BP has a specific solution for which $\theta=\theta_{c}$ for all $s \in \mathbb{R}$. It is called the homothetic solution because the ratio $y / x=\tan \theta_{c}$ remains constant (see [17]). It is seen as a segment in the configuration plane $\mathcal{U}$ and divides it into two regions: the region of the $\mathrm{DBC}$ for $\theta \in\left[\theta_{\alpha}, \theta_{c}\right)$, and the region of the SBC for $\theta \in\left(\theta_{c}, \pi / 2\right]$. See Figure 2 .

Both equilibrium points are hyperbolic: the differential of the vector field 175 evaluated at the equilibrium points has four different real eigenvalues, two positive and two negative, for any value of $\alpha$, see Lemma 1 in the Appendix. Also, we give explicit formulas for the eigenvalues and the corresponding eigenvectors in terms of $\alpha, \theta_{c}$ and the energy $h$.

Therefore, there exist the corresponding stable and unstable invariant manifolds $W^{s}\left(E^{ \pm}\right)$and $W^{u}\left(E^{ \pm}\right)$. On the constant energy manifold their dimensions are the following:

$$
\operatorname{dim}\left(W^{u}\left(E^{+}\right)\right)=\operatorname{dim}\left(W^{s}\left(E^{-}\right)\right)=2, \quad \operatorname{dim}\left(W^{u}\left(E^{-}\right)\right)=\operatorname{dim}\left(W^{s}\left(E^{+}\right)\right)=1 .
$$

In particular, the invariant manifolds $W^{u}\left(E^{-}\right)$and $W^{s}\left(E^{+}\right)$are embedded in the total collision manifold $\mathcal{C}$. In Figure 3 , we plot their projection in the $(\theta, v)$ plane for two values of the mass parameter $\alpha$.

The dynamics on the total collision manifold $\mathcal{C}$ is the key to understanding the solutions of the SC4BP that go close to quadruple collision, in particular, the ejection-collision orbits. In [2, Simó and Lacomba show that there exists 185 a sequence of values $\left\{\alpha_{k}\right\}_{k \geq 1}$ for which one or both branches of $W^{u}\left(E^{-}\right)$and $W^{s}\left(E^{+}\right)$coincide (a single or double heteroclinic connection). For values of $\alpha \in\left(\alpha_{k}, \alpha_{k+1}\right)$ the branches of these invariant manifolds (that are contained in 

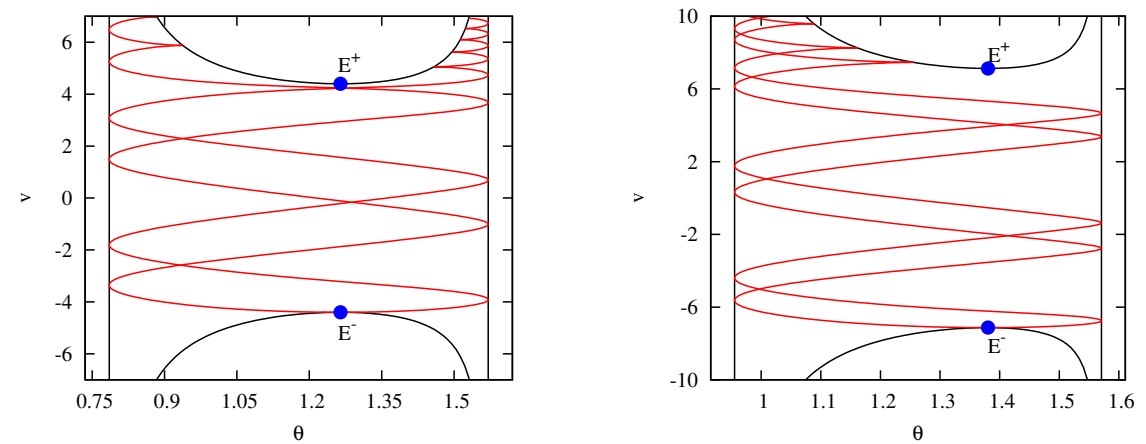

Figure 3: Projection on the $(\theta, v)$ plane of the total collision manifold $\mathcal{C}$ and the two branches of the invariant manifold $W^{u}\left(E^{-}\right)$for $\alpha=1$ (left) and $\alpha=2$ (right). $W^{s}\left(E^{+}\right)$can be obtained using the symmetric solution 9 .

$\mathcal{C})$, behave similarly in a topological sense: first they have a number of alternating double collisions of both types, and then only perform one type of binary collision as $v$ increases. The number and type of binary collisions of each branch of $W^{u}\left(E^{-}\right)$(similarly for $\left.W^{s}\left(E^{+}\right)\right)$are the same for any $\alpha \in\left(\alpha_{k}, \alpha_{k+1}\right)$. In Figure 3 we show one example where each branch of $W^{u}\left(E^{-}\right)$performs different types of binary collisions as $v$ increases (left, case $\alpha=1 \in\left(\alpha_{3}, \alpha_{4}\right)$ ) and another example where both branches perform the same type of binary collisions (right, case $\left.\alpha=2 \in\left(\alpha_{4}, \alpha_{5}\right)\right)$. That behavior has been used by Lacomba and Medina in [20] to prove the existence of some ejection-collision orbits for specific intervals of values of $\alpha$ depending on the behavior of the invariant manifold $W^{u}\left(E^{-}\right)$.

Our purpose in this paper is to give a numerical general methodology that allows to compute the ejection-collision orbits for any value of $\alpha$. Clearly, these orbits belong to the intersection of the invariant manifolds $W^{u}\left(E^{+}\right)$and $W^{s}\left(E^{-}\right)$ in $\mathbb{R}^{4}$. Thus, in order to deal with them, we construct an approximation of their parametrizations. The approximations of order one are given by

$$
\begin{aligned}
& \Psi_{1}^{-}(\xi, \varphi)=E^{-}+\xi\left(\cos (2 \pi \varphi) \bar{\sigma}_{1}+\sin (2 \pi \varphi) \bar{\sigma}_{4}\right), \\
& \Psi_{1}^{+}(\xi, \varphi)=E^{+}+\xi\left(\cos (2 \pi \varphi) \bar{\sigma}_{1}+\sin (2 \pi \varphi) \bar{\sigma}_{3}\right)
\end{aligned}
$$

where $\bar{\sigma}_{i}$ are the corresponding eigenvectors and $\xi>0$ is a small fixed quantity, the distance from the initial conditions to the equilibrium point. See the Ap- 
pendix for more details and (24) for the specific expressions of the first order approximation for each variable. In fact, using the symmetry $(9)$, for any orbit $\Gamma \subset W^{s}\left(E^{-}\right)$, we have that $\bar{\Gamma} \subset W^{u}\left(E^{+}\right)$, and the other way around. Therefore, it is enough to construct the parametrization of one of the invariant manifolds.

From the particular expressions for the variables $r$ and $\theta$ (see $(24)$ in the Appendix) we make two remarks. On one hand, we have that $r>0$ only for values $\varphi \in(1 / 4,3 / 4)$, so these will be the only values considered. The values $\varphi=1 / 4,3 / 4$ give the parametrization of the orbits of the invariant manifold inside the collision manifold $\mathcal{C}$. On the other hand, the values $\varphi \in(1 / 4,1 / 2)$ are such that the initial $\theta$ satisfies $\theta>\theta_{c}$, so the orbits start in the region of the $\mathrm{SBC}$, whereas values $\varphi \in(1 / 2,3 / 4)$ correspond to initial values $\theta<\theta_{c}$, so the orbits start in the region of the DBC. The homothetic solution is obtained when $\varphi=1 / 2$.

The vectors $\bar{\sigma}_{1}$ and $\bar{\sigma}_{3,4}$ give the slow and fast directions, respectively, of the linear dynamics on $W^{u}\left(E^{+}\right), W^{s}\left(E^{-}\right)$, so the slow and fast submanifolds 215 are given by $\varphi=1 / 2$ and $\varphi=1 / 4,3 / 4$. The orbits close to the slow direction, which here coincides with the homothetic solution, will be difficult to follow due to the stronger pull of the fast direction. In practice this means that in order to consider orbits close to the homothetic solution, we need to take values $\varphi \in(1 / 2-\varepsilon, 1 / 2+\varepsilon)$, for $\varepsilon$ small enough. As we will show, the richness of the dynamics on the invariant manifold (in the sense of greater variety of orbits exhibiting different numbers of binary collisions, and in particular to obtain ejection-collision orbits) occurs precisely around the homothetic solution. For example, taking $\alpha=1$ and $h=-1$, for most of the values $\varphi \in(1 / 4,3 / 4)$, the orbits escape to infinity directly exhibiting only one type of binary collisions (see 225 Section 33, so they are useless in order to compute ejection-collision orbits. If we take the approximation of order one, $\Psi_{1}^{ \pm}(\xi, \varphi)$, to work with a good precision, for example of order $10^{-12}$ (see below for details), it will be necessary to consider values of $\xi$ less than $10^{-6}$. However, with such small values of $\xi$, in order to show the richness of the dynamics around the homothetic we need to consider values $\varepsilon \simeq 10^{-9}$. 
Therefore, if we want to consider initial conditions close to the slow direction, we need to start farther away from the equilibrium point, that is, with bigger values of $\xi$. Following [19] (specifically, Chapters 1 and 2), we derive the parametrization of the invariant manifold up to different orders $\Psi_{m}^{+}$, for $m \leq 8$, and we have performed several numerical tests to control the quality of the approximation. Specifically, one can compute how big $\xi$ can be in order to maintain a certain accuracy. To do that, for each order $m$ and distance $\xi$, compute the error in the orbit $e_{o}(s, \xi, \varphi)$ (see Section 2.5 of [19]) for $s \in[0,1]$ and the maximum value over all the orbits

$$
E_{o}(\xi)=\max _{\varphi \in(1 / 4,3 / 4)} e_{o}(1, \xi, \varphi) .
$$

The same procedure can be applied to the errors in the invariance equation and the errors in the energy (see [19] for details). For $\alpha=1$ and $h=-1$ we have computed $E_{o}(\xi)$ and the parametrizations $\Psi_{m}^{+}$for $m=2,3,5,8$, see Figure 4 For example, in order to have an accuracy below $10^{-8}$ with $m=5$ we need $\xi$ 235 up to 0.015 approximately, whereas with $m=8$ we can take values up to order 0.05 .

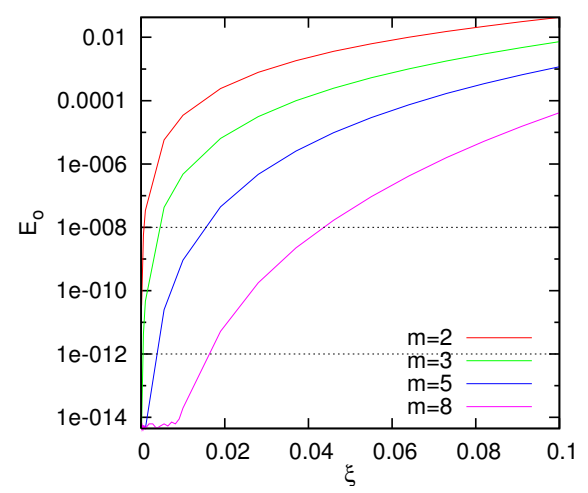

Figure 4: Error of the approximations of the parametrizations $\Psi_{m}$ for $m=2,3,5,8$ with respect the distance $\xi$ at which the initial conditions on $W^{u}\left(E^{+}\right)$are taken, for $\alpha=1$ and $h=-1$.

For the purposes of this work, we have considered, for $\alpha=1$, a parametrization of order $m=8$ and $\xi$ of order $10^{-2}$. With these values, we will show that 
all the ejection-collision trajectories will be found for $\varphi \in(1 / 2-\varepsilon, 1 / 2+\varepsilon)$, for

\subsection{Poincaré section}

A common tool, in order to study the dynamics of a problem given by an autonomous system of differential equations, is the Poincaré map, which is defined on a surface of section. In [12, Sekiguchi and Tanikawa perform a wide exploration of the dynamics of the problem using as a surface of section $\left\{\theta=\theta_{c}\right\}$. In fact, an orbit cannot cross the section $\left\{\theta=\theta_{c}\right\}$ between two consecutive binary collisions of the same type (see Theorem 7 in [12]).

At this point we want to stress the attention on two important facts for the computation of ejection-collision orbits. These orbits start and end at $r=$ 0 , which corresponds to the boundary of the section $\left\{\theta=\theta_{c}\right\}$ in the $(v, w)$ plane, used by Sekiguchi and Tanikawa [12. In their representations of the return map to that section, different ejection-collision orbits, that are on that boundary, share common points. Therefore, they are indistinguishable in that representation. Moreover, the authors assure in Theorem 1 that all the solutions of the SC4BP must cross the section $\left\{\theta=\theta_{c}\right\}$ forwards or backwards in time at least once. That is not true. In the proof the authors forget about the invariant manifolds $W^{u}\left(E^{+}\right)$and $W^{s}\left(E^{-}\right)$. We will show in Section 3 that there exist orbits that start at a quadruple collision and escape directly without crossing $\left\{\theta=\theta_{c}\right\}$ (see Theorem 1 and in Section 4 we will show ejection-collision orbits that do not cross that section (by cross we always mean transversal intersection).

Taking into account that, typically, all the orbits perform binary collisions (with the exception of the homothetic orbit), we consider the section

$$
\Sigma_{c}=\left\{(r, v, \theta, w) ; w=0, \theta=\theta_{\alpha} \text { or } \theta=\pi / 2\right\}
$$

which corresponds to both types of binary collisions: SBC and DBC. 


\section{Escape orbits}

Notice that there are two different ways for the particles to escape to infinity. In one case, the outer bodies escape, while the inner bodies perform consecutive single binary collisions -escape of type 1-. In the other case, all the bodies escape performing consecutive double binary collisions - escape of type $2-$.

Definition 2. A solution of the $S C 4 B P$ is an escape orbit if some or all particles go to infinity forwards or backwards in time. More concretely, it has an escape of type 1 if $y \rightarrow+\infty$ while $x$ remains bounded, whereas it has an escape of type 2 if $x, y \rightarrow+\infty$.

Notice that when the escape is of type 1 , the solution stays in the region $\theta>\theta_{c}$ when $s \rightarrow \pm \infty$ (forwards/backwards), so only SBC occur (collisions of type 1). Similarly, when the escape is of type 2 , the solution stays in the region $\theta_{\alpha}<\theta<\theta_{c}$ when $s \rightarrow \pm \infty$ (forwards/backwards), so only DBC occur 275 (collisions of type 2).

Sekiguchi and Tanikawa [12] (Theorem 5) established analytical sufficient conditions to determine when an orbit is an escape orbit. We follow the same arguments to derive the same criteria in our variables.

Proposition 1. Let $P=(r, v, \theta, w)$ be a point in the phase space $\mathcal{F}, \gamma(s)$ be a solution of the equations (6) that goes through $P$, and $\delta=\tan \left(\theta_{c}\right)$ (given in (10)).

1. For $\theta>\theta_{c}$, let $\Upsilon=v \sin \theta+\frac{w}{\Delta(\theta)} \cos \theta$. If

$$
\sin \theta \Upsilon^{2} \geq \alpha^{3 / 2} \sqrt{2}\left(\alpha+8 \delta^{2} \frac{\delta^{2}+\alpha}{\left(\delta^{2}-\alpha\right)^{2}}\right),
$$

then the orbit has an escape of type 1.

2. For $\theta<\theta_{c}$, let $\Upsilon=-w \sqrt{W(\theta)}+v \cos ^{2} \theta+v \sqrt{\alpha} \cos \theta \sin \theta$. If

$$
\begin{aligned}
\sec \theta(1+\sqrt{\alpha} \tan \theta) \Upsilon^{2} \geq(1+\alpha)^{2} \sqrt{2} \\
\left(\frac{1}{(1-\sqrt{\alpha} \varepsilon)^{2}}+\frac{\alpha^{3}}{(\sqrt{\alpha}+\varepsilon)^{2}}+\frac{8 \alpha^{2}}{(2 \sqrt{\alpha}+(1-\alpha) \varepsilon)^{2}}\right),
\end{aligned}
$$

where $\varepsilon=(\delta-\sqrt{\alpha}) /(1+\delta \sqrt{\alpha})$, then the orbit has an escape of type 2. 
In both cases, the orbit escapes forwards or backwards in time depending on whether $\Upsilon$ is positive or negative, respectively.

Proof. We follow exactly the same methodology as in [12] using our notation.

Consider the first case $\theta>\theta_{c}$ (in the configuration plane, the region of SBC, where $x$ is bounded). We want to see that if the sufficient condition is satisfied, then $\lim _{s \rightarrow \infty} y(s)=\infty$. From the system of equations associated to the Hamiltonian (1), the equation for the variable $y$ writes

$$
\ddot{y}=-\frac{1}{y^{2}} f(x / y)
$$

where $f(z)=\frac{\alpha^{5 / 2}}{4}+2 \alpha^{3 / 2} \frac{1+\alpha z^{2}}{\left(1-\alpha z^{2}\right)^{2}}$ is a positive monotone increasing function for $z \in(0,1 / \sqrt{\alpha})$. The region $\theta>\theta_{c}$ corresponds to $z=x / y<1 / \delta$ where $\delta=\tan \left(\theta_{c}\right)$ and

$$
\ddot{y} \geq-\frac{f(1 / \delta)}{y^{2}} .
$$

It means that the force acting on the external bodies is bounded from below by the force of the two-body problem given by $-\frac{f(1 / \delta)}{y^{2}}$. Thus, a sufficient condition for escape motion is that the total energy of the two-body problem should be to be positive, that is $y \dot{y}^{2} \geq 2 f(1 / \delta)$. In our variables $(\theta, v, u)$ it becomes

$$
\sin \theta(v \sin \theta+u \cos \theta)^{2} \geq 4 \sqrt{2} f(1 / \delta) .
$$

Now, if we rewrite (13) in terms of the variables $(\theta, v, w)$, the result stated in the item 1. of the proposition is followed.

The next is to consider the case with $\theta<\theta_{c}$, which corresponds to the region $\mathrm{DBC}$ in the configuration plane. In this hypothesis, escape motion means that the four masses escape to infinity. Therefore, both $x, y \rightarrow \infty$ and we need to introduce Jacobi variables:

$$
Q_{1}=x+\sqrt{\alpha} y, \quad Q_{2}=y-\sqrt{\alpha} x .
$$

That is, essentially, $Q_{1}$ is the center of mass and $Q_{2}$ is the distance between $m_{2}$ and $m_{4}$. In these variables, the escape through the $\mathrm{DBC}$ region corresponds to 
$Q_{1} \rightarrow \infty$ whereas $Q_{2}$ remains bounded. The equation of motion corresponding to $Q_{1}$ becomes

$$
\ddot{Q}_{1}=\frac{-(1+\alpha)^{2}}{Q_{1}^{2}} g\left(Q_{2} / Q_{1}\right)
$$

where $g(z)=\frac{1}{4(1-\sqrt{\alpha} z)^{2}}+\frac{\alpha^{3}}{4(\sqrt{\alpha}+z)^{2}}+\frac{2 \alpha^{2}}{(2 \sqrt{\alpha}+(1-\alpha) z)^{2}}$ is a positive monotone increasing function for $z \in(0,1 / \sqrt{\alpha})$. Therefore, when $\theta<\theta_{c}$, we have $y / x<\delta$ and

$$
\ddot{Q}_{1} \geq \frac{-(1+\alpha)^{2}}{Q_{1}^{2}} g(D)
$$

for $z \leq D=(\delta-\sqrt{\alpha}) /(1+\delta \sqrt{\alpha})$. Again, a sufficient condition for escape motion is the total energy of the two-body problem must be positive. It means that

$$
Q_{1} \dot{Q}_{1}^{2} \geq 2(1+\alpha)^{2} G(D)
$$

As in the previous case, if we rewrite 14 in terms of the variables $(\theta, v, w)$, the result stated in the item 2 . of the proposition is followed.

Next, we use the above criteria and the linear approximation of the parametrization of the invariant manifolds to show that there are orbits that escape forwards (or backwards) in time and have only one type of binary collision. We call them ejection-direct escape orbits of type 1 or type 2 depending on the type of the escape. In particular, this result shows that there exist solutions of the SC4BP that do not cross the section $\left\{\theta=\theta_{c}\right\}$ (see Section 2.3).

Theorem 1. There exist ejection-direct escape orbits of type 1 and of type 2, that is, orbits starting (or ending) at the quadruple collision and escaping to (coming from) infinity with binary collisions only of one type. orbits on $W^{u}\left(E^{+}\right)$that escape forwards in time performing binary collisions only of one type. We will prove the result for orbits that have only SBC. The proof to obtain orbits with only DBC is similar.

We will use the criteria given in Proposition 1. As we want to prove escape 
forwards in time of type one, we must see that

$$
\begin{aligned}
& F(v, \theta, w)=\Upsilon^{2} \sin \theta-\alpha^{3 / 2} \sqrt{2}\left(\alpha+8 \delta^{2} \frac{\delta^{2}+\alpha}{\left(\delta^{2}-\alpha\right)^{2}}\right) \geq 0, \\
& \Upsilon(v, \theta, w)=v \sin \theta+w \cos \theta / \Delta(\theta)>0 .
\end{aligned}
$$

Using the approximation of the parametrization of order 1 of the invariant manifold $\Psi_{1}^{+}(\xi, \varphi)$ given in 11 and 24 , and omitting the variable $r$, a point on $W^{u}\left(E^{+}\right)$close to the equilibrium point can be written, for $\xi$ small enough, as

$$
p=(v, \theta, w)=p_{0}+p_{1}+O\left(\xi^{2}\right),
$$

where $p_{0}=\left(v_{c}, \theta_{c}, 0\right)$ and $p_{1}$ are the terms of order 1 in $\xi$ for values $\varphi \in$ $(1 / 4,1 / 2)$, so that we ensure that $\theta>\theta_{c}$ and the initial condition is in the region of $\mathrm{SBC}$.

Clearly $\Upsilon(p)=\Upsilon\left(p_{0}\right)+O(\xi)=v_{c} \sin \theta_{c}+O(\xi)>0$ for $\xi$ small enough. Next, consider $F(p)=F\left(p_{0}\right)+D F\left(p_{0}\right) \cdot p_{1}+O\left(\xi^{2}\right)$. On one hand, $\Upsilon\left(p_{0}\right)^{2} \sin \theta_{c}=$ $2 V\left(\theta_{c}\right) \sin ^{3} \theta_{c}$, where $V(\theta)$ is the potential function given in (4). Using that $V^{\prime}\left(\theta_{c}\right)=0$, after some computations, one can get that $F\left(p_{0}\right)=0$. On the other hand,

$$
D F\left(p_{0}\right)=2 v_{c} \sin ^{2} \theta_{c}\left(\sin \theta_{c}, \frac{3 v_{c}}{2} \cos \theta_{c}, \frac{\cos \theta_{c}}{\Delta\left(\theta_{c}\right)}\right),
$$

and using 24

$$
\begin{aligned}
D F\left(p_{0}\right) \cdot p_{1}=2 \xi v_{c} \sin ^{2} \theta_{c}\left(\frac{-h \sin \theta_{c}}{\sqrt{h^{2}+v_{c}^{2}}} \cos (2 \pi \varphi)\right. & \\
& \left.\quad+\frac{\cos \theta_{c}}{\sqrt{1+\lambda_{3}^{2}}}\left(\frac{3 v_{c}}{2}+\frac{\lambda_{3}}{\Delta\left(\theta_{c}\right)}\right) \sin (2 \pi \varphi)\right)
\end{aligned}
$$

where $h<0, \Delta\left(\theta_{c}\right)>0$ (see (5) ) and $\lambda_{3}=\lambda_{3}\left(E^{+}\right)>0$. Therefore, if we consider values $\varphi=1 / 4+\varepsilon, D F\left(p_{0}\right) \cdot p_{1}>0$, which concludes the proof.

The proof of Theorem 1 shows that the orbits with initial conditions $\Psi_{1}^{ \pm}(\xi, \varphi)$ with $\varphi=1 / 4+\varepsilon$ are the ones that escape (forwards/backwards) directly to infinity exhibiting only $\mathrm{SBC}$ (type 1) (analogous with $\varphi=3 / 4-\varepsilon$ and DBC). Recall that the values $\varphi=1 / 4,3 / 4$ correspond to the fast direction on the 
ariant manifold, whereas $\varphi=1 / 2$ corresponds to the homothetic and the slow direction. Therefore, the farther an initial condition from the homothetic solution is, the higher the probability to escape directly to infinity.

We show numerically, for $\alpha=-1$ and $h=-1$, that the orbits with initial conditions $\Psi_{8}^{+}(\xi, \varphi)$ for $\varphi=1 / 4+\varepsilon$ and $\varphi=3 / 4-\varepsilon$ are the ones that escape directly. We consider the unstable manifold $W^{u}\left(E^{+}\right), \xi=10^{-2}$ and vary $\varphi \in$ $(1 / 4,3 / 4)$. Given an initial condition, we integrate the ODE (6) forwards in time and, at each step, we control the escape condition. If the condition is satisfied and the orbit has not crossed the section $\left\{\theta=\theta_{c}\right\}$, we save the time $s_{e}$ at that point. In Figure 5 we show, on the left, that for all values of $\varphi \in(1 / 4,0.49998)$

all the orbits escape directly with collisions only of type 1. A similar behavior is shown for $\varphi \in(0.500006,3 / 4)$ (right plot), with orbits escaping directly with collisions only of type 2. In Figure 5, some examples of direct escape are plotted for $s \leq s_{e}$.

\section{Ejection-collision orbits}

In this Section we present some results about the ejection-collision orbits, as well as the methodology to compute and classify them. Also the results obtained for $\alpha=1$ are presented.

Definition 3. An ejection-collision orbit (ECO) of the $S C 4 B P$ is a solution $\Gamma=\{\gamma(s)\}_{s \in \mathbb{R}}$ of (6) such that $\lim _{s \rightarrow \pm \infty} r(s)=0$.

An ECO is a solution that starts and ends in a quadruple collision. Therefore, the orbit belongs to the intersection $W^{u}\left(E^{+}\right) \cap W^{s}\left(E^{-}\right)$. More concretely,

$$
\lim _{s \rightarrow-\infty} \gamma(s)=E^{+}, \quad \lim _{s \rightarrow+\infty} \gamma(s)=E^{-},
$$

it is a heteroclinic connection between the two equilibrium points.

Using the symmetry of the problem given by (8), we can prove the following statements:

Proposition 2. Let $\Gamma$ be a solution of the $S C 4 B P$. 

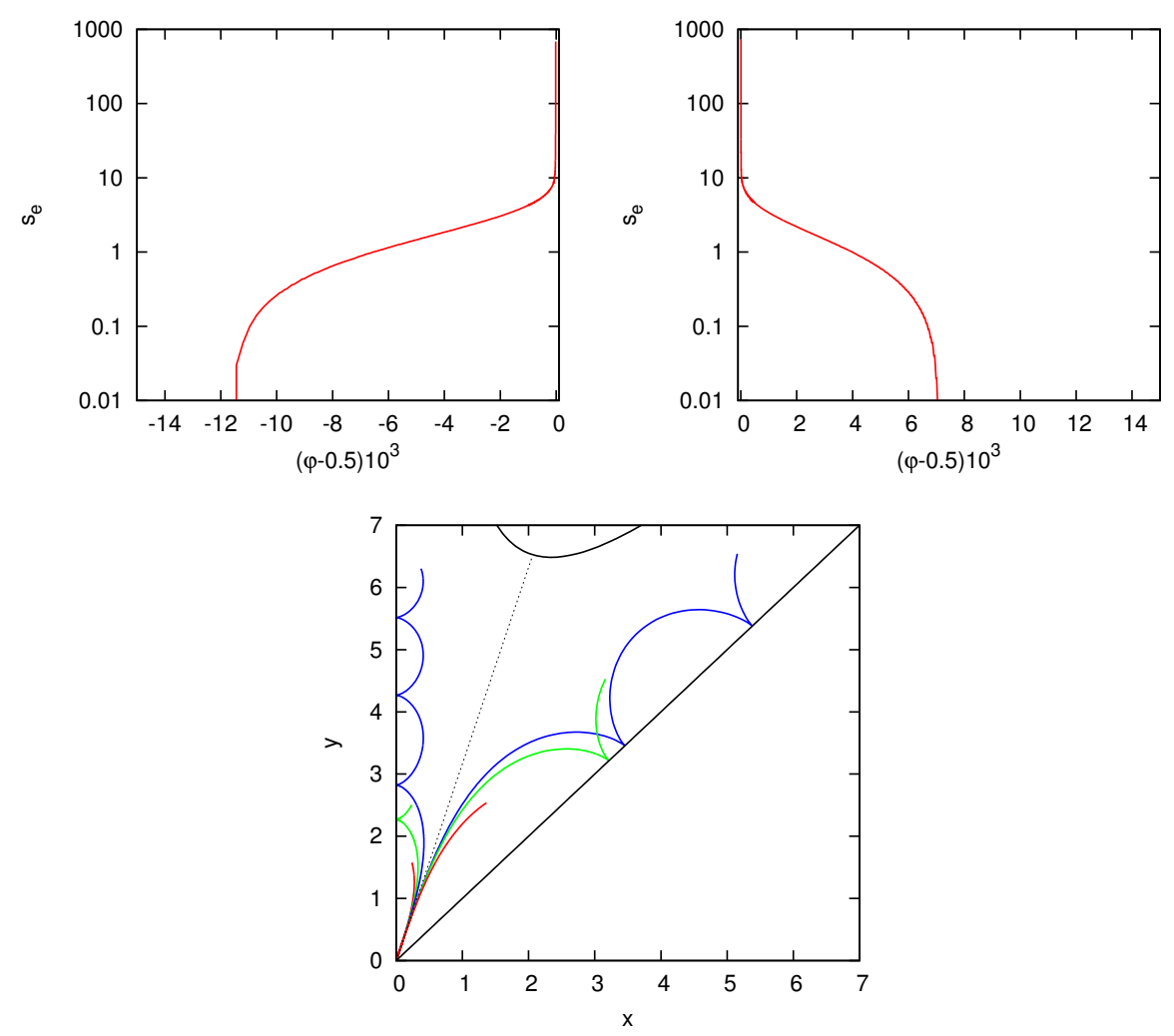

Figure 5: For orbits on $W^{u}\left(E^{+}\right)$with initial conditions $\Psi_{8}^{+}(0.01, \varphi)$ for $\alpha=1$ and $h=-1$, we plot the time $s_{e}$ (in logarithmic scale) at which the escape condition through the SCB (top left plot) or DCB (top right plot) regions is satisfied without crossing $\theta=\theta_{c}$. Bottom: some example of orbits of direct escape, for $s \leq s_{e}$.

1. If $\Gamma \in W^{u}\left(E^{+}\right)$is a symmetric solution (see Definition 1), then it is an ECO.

2. If $\Gamma$ is an ECO, then $\bar{\Gamma}$ (defined in (9)) is also an ECO.

Proof. By definition, if $\Gamma \in W^{u}\left(E^{+}\right)$, then $\lim _{s \rightarrow-\infty} r(s)=0$. If the solution is symmetric, then $\lim _{s \rightarrow+\infty} r(s)=0$, so it is an ECO.

If $\Gamma=\{\gamma(s)\}_{s \in \mathbb{R}}$ is an ECO, then it connects $E^{+} \longrightarrow E^{-}$. Then, by definition of $\bar{\gamma}(s)$

$$
\lim _{s \rightarrow-\infty} \bar{\gamma}(s)=E^{+}, \quad \lim _{s \rightarrow+\infty} \bar{\gamma}(s)=E^{-}
$$


Therefore $\bar{\Gamma}$ is an ECO.

If $\Gamma$ is an ECO, both $\Gamma$ and $\bar{\Gamma}$ trace the same path in the configuration space $(r, \theta)($ or $(x, y))$ in opposite sense.

Notice that, if a solution $\Gamma=\{\gamma(s)\}_{s \in \mathbb{R}} \in W^{u}\left(E^{+}\right)$, then $\lim _{s \rightarrow-\infty} \theta(s)=$ $\theta_{c}$. Therefore, $\Gamma$ only can have a finite number of binary collisions backwards in time, so there exists a first binary collision. Recall that the binary collisions can be viewed as the intersections of a solution with the section $\Sigma_{c}$. We denote

$$
p_{j}=\left\{\begin{array}{l}
1 \text { the } j \text {-th intersection is a } \mathrm{SBC}, \\
2 \text { the } j \text {-th intersection is a DBC, }
\end{array} \text { for } j=1, \ldots, n .\right.
$$

Let $B$ be the set of all possible sequences just taking into account the elements 1 and 2. Thus, we can define

$$
\begin{aligned}
P: W^{u}\left(E^{+}\right) & \longrightarrow B \\
\Gamma & \longrightarrow\left(p_{1}, p_{2}, \ldots, p_{n}, \ldots\right)
\end{aligned}
$$

345 For example, the escape orbits shown in Figure 5 satisfy $P(\Gamma)=(1,1, \ldots)$ or $P(\Gamma)=(2,2, \ldots)$, that is, they only exhibit the same type of binary collision. In Figure 6 we show the orbits $\Gamma_{1}$ and $\Gamma_{2}$ such that $P\left(\Gamma_{1}\right)=(1,1, \ldots)$ and $P\left(\Gamma_{2}\right)=(1,2, \ldots)$.

Using the map $P$ we have the following characterizations and properties.

Proposition 3. Let $\Gamma \in W^{u}\left(E^{+}\right)$. Then

1. $P(\Gamma)$ is finite if and only if it corresponds to an ECO.

2. If $\Gamma$ is an ECO such that $P(\Gamma)=\left(p_{1}, p_{2}, \ldots, p_{n}\right)$, then $\bar{\Gamma}$ is an ECO with $P(\bar{\Gamma})=\left(p_{n}, p_{n-1}, \ldots, p_{1}\right)$.

3. $\Gamma$ is a symmetric ECO if and only if $P(\Gamma)$ is a symmetric sequence.

Proof. Suppose that an ECO has infinity binary collisions. Then, there exists a sequence $S=\left\{s_{n}\right\}_{n \geq 1}$ such that $\theta\left(s_{n}\right) \in\left\{\theta_{\alpha}, \pi / 2\right\}$. Because the orbit is an ECO, $\lim _{s \rightarrow \pm \infty} \theta(s)=\theta_{c}$, and $S$ must be bounded which is not possible as the function $\theta(s)$ is analytic. The second statement comes from the definition of a symmetric solution $\bar{\Gamma}$. The last one is clear using that $P(\Gamma)=P(\bar{\Gamma})$. 


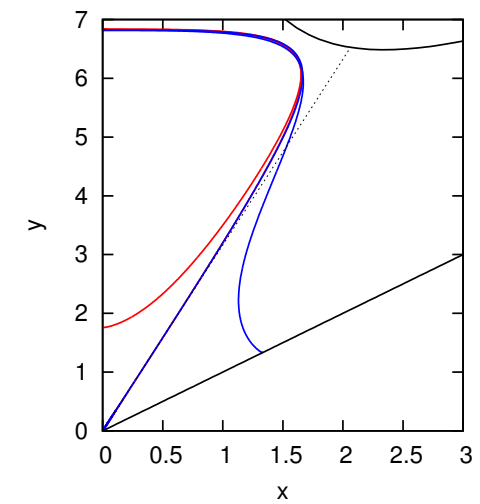

Figure 6: Two orbits $\Gamma_{1,2} \in W^{u}\left(E^{+}\right)$plotted up to their second intersection with the section $\Sigma_{c}$. In one case, the two intersections correspond to $\mathrm{SBC}$, so $P\left(\Gamma_{1}\right)=(1,1, \ldots)$. In the other case, the two intersections correspond to binary collision of different type with $P\left(\Gamma_{2}\right)=$ $(1,2, \ldots)$.

Definition 4. $\Gamma$ is an ECO of order $n$ if $P(\Gamma)=\left(p_{1}, p_{2}, \ldots, p_{n}\right)$.

If an ECO of order $n$ is symmetric, depending on the parity of $n$, it must touch the zero velocity curve.

Proposition 4. Let $\Gamma$ be a symmetric ECO of order $n$. Then, the orbit has a point on the zero velocity curve if and only if $n$ is even. In this case, such point takes place between the $(n / 2)$-th and $(n / 2+1)$-th binary collision.

Proof. If $\Gamma=\{\gamma(s)\}_{s \in \mathbb{R}}$ is symmetric, then there exists a time $s_{0}$ such that the orbit traces the same trajectory in the $(r, \theta)$ plane before and after $s_{0}$. Therefore, $\gamma\left(s_{0}\right)$ is a point of return and the number and type of binary collisions must be the same before and after $s_{0}$. Thus, $n$ must be even and the point must be on the zero velocity curve.

The dynamics on the total collision manifold $\mathcal{C}$ is the key to prove the existence of ECO. In [20, the authors use that information to show the existence of some ECO for specific values of $\alpha$. In particular, they prove that for any value of the mass parameter $\alpha$ and for any natural number $n$ there exists an ECO exhibiting only and exactly $n$ SBC or $n$ DBC. They also prove that for 
$\alpha \in\left(\alpha_{3}, \alpha_{4}\right)$ (see Section 2.2), an ECO with $P(\Gamma)=(1,2,1,2,1)$ exists (in particular this is true for $\alpha=1$ ).

Our aim is to present a methodology to compute and classify the ECO for any fixed value of $\alpha$. We will show and analyze the results obtained for the specific value $\alpha=1$.

\subsection{Methodology}

In order to look for heteroclinic connections between the two equilibrium points, the main idea is to analyze the successive intersections of the orbits of the invariant manifolds $W^{s}\left(E^{-}\right)$and $W^{u}\left(E^{+}\right)$with the section $\Sigma_{c}$, defined in 12. Due to the symmetry of the problem, it is enough to deal with one of the invariant manifolds. In what follows, we consider initial conditions on $W^{u}\left(E^{+}\right)$ and the approximation of its parametrization, $\Psi_{m}^{+}(\xi, \varphi)$, for a suitable $m$ and for a fixed value of $\xi$. For simplicity we denote the parametrization simply by $\Psi(\varphi)$. Then, each orbit $\gamma(s) \in W^{u}\left(E^{+}\right)$is characterized by its initial condition given by

$$
\gamma(0)=\Psi(\varphi)=\left(r_{0}, v_{0}, \theta_{0}, w_{0}\right)
$$

where $\varphi \in(1 / 4,3 / 4)$. For each $\varphi$, we integrate forward in time to compute the first $n$ intersections of $\gamma(s)$ with $\Sigma_{c}$. We define the map

$$
\begin{aligned}
P_{n}:(1 / 4,3 / 4) & \longrightarrow B \\
\varphi & \longrightarrow\left(p_{1}, p_{2}, \ldots, p_{n}\right),
\end{aligned}
$$

where $P_{n}(\varphi)=\left(p_{1}, p_{2}, \ldots, p_{n}\right)$ codes the first $n$ intersections with $\Sigma_{c}$ of the solution $\gamma(s)$ with initial condition $\Psi(\varphi)$.

We want to notice here that for a given $\varphi, p_{1}$ is the first binary collision after the initial condition. Depending on the the initial distance $\xi$ considered, there could exist a binary collision before the initial condition (backwards in time, towards the quadruple collision). This is specially true for values of $\varphi$ near to $1 / 4$ and $3 / 4$. As we explained in Section 3 , the solutions corresponding to values far from $\varphi=1 / 2$ (the homothetic orbit) escape directly to infinity. So the ECO will be found for values $\varphi \in(1 / 2-\varepsilon, 1 / 2+\varepsilon)$, for $\varepsilon$ small, depending 
on $\alpha$ and $\xi$. For example, for $\alpha=1$ and $\xi$ of order $10^{-2}, \varepsilon \simeq 10^{-4}$. We will show that for such values of $\varphi$, and for the order of the ECO computed, the first binary collision takes place far away from the equilibrium point, so $P_{n}(\varphi)$ starts with the very first binary collision of the orbit.

Up to now, we have characterized the ejection-collisions orbits in terms of the map $P$. In order to detect and compute them we use the following result:

Proposition 5. Let the maps $P$ and $P_{n}$ be given in (16) and (17), and $\varphi_{1}<\varphi_{2}$ be such that

$$
\begin{aligned}
& P_{n+1}\left(\varphi_{1}\right)=\left(p_{1}, p_{2}, \ldots, p_{n}, p_{n+1}^{1}\right) \\
& P_{n+1}\left(\varphi_{2}\right)=\left(p_{1}, p_{2}, \ldots, p_{n}, p_{n+1}^{2}\right)
\end{aligned}
$$

with $p_{n+1}^{1} \neq p_{n+1}^{2}$. Then, there exists a value $\varphi \in\left(\varphi_{1}, \varphi_{2}\right)$ such that the solution $\Gamma$ with initial condition $\Psi(\varphi)$ is such that $P(\Gamma)=\left(p_{1}, p_{2}, \ldots, p_{n}\right)$.

The proof is straightforward by continuity with respect to the initial condition $\varphi$.

An illustration of Proposition 5 is shown in Figure 6, the red orbit $\Gamma_{1}$ has an initial condition $\Psi\left(\varphi_{1}\right)$ with $P_{2}\left(\varphi_{1}\right)=(1,1)$ and the blue orbit $\Gamma_{2}$ has an initial condition $\Psi\left(\varphi_{2}\right)$ with $P_{2}\left(\varphi_{2}\right)=(1,2)$. So, in between there exists an ECO such that $P(\Gamma)=(1)$.

Using Proposition 5 to detect the existence of an ECO of order $n$, we vary $\varphi \in(1 / 4,3 / 4)$ and we compute $P_{n+1}(\varphi)$ integrating the equations 6 of the SC4BP up to the $(n+1)$-th crossing with $\Sigma_{c}$. To detect a change in the type of the binary collision, it is enough to track the value of $\theta$ at the $(n+1)$-th crossing, $\theta_{n+1}(\varphi)$ : when it changes from $\pi / 2$ to $\theta_{\alpha}$, or the other way around, we are in the situation of Proposition 5. Although the discontinuities of the function $\theta_{n+1}(\varphi)$ show the existence of ECO, we propose to use, instead, the function $F_{n+1}(\varphi)=r\left(\theta-\theta_{c}\right)$, where $r$ and $\theta$ are the values of the orbit at the $(n+1)$-th 415 intersection with the section $\Sigma_{c}$ (or the $(n+1)$-th binary collision). Clearly, the function $F_{n+1}$ is continuous and due to the fact that $r>0$, it changes sign depending on whether $\theta$ is greater or smaller that $\theta_{c}$. Therefore, each solution 
of $F_{n+1}(\varphi)=0$ corresponds to an ECO orbit of order $j \leq n$. We track the sign of the function $F_{n+1}$ and apply an iterative method to obtain the value of $\varphi$ (up to a certain precision) that corresponds to the ECO. We have repeated the explorations for $\alpha=1$ and different values of $\xi=0.001,0.01,0.05$. In all cases we have obtained the same results (that is, the same ECO). In Figure 7 we show the values of $\theta_{n+1}(\varphi)$ and $F_{n+1}(\varphi)$ for $n=4,5,6$ and $\varphi<1 / 2$, using $\alpha=1, \xi=0.05$ and $h=-1$. For $n=4$ the function $F_{5}$ shows four zeros, corresponding to four ECO of order $n \leq 4$ (see Table 1 in next section), for $n=5$, the function $F_{6}$ shows six zeros, corresponding to the same ECO and two new ones, of order 5. And so on.
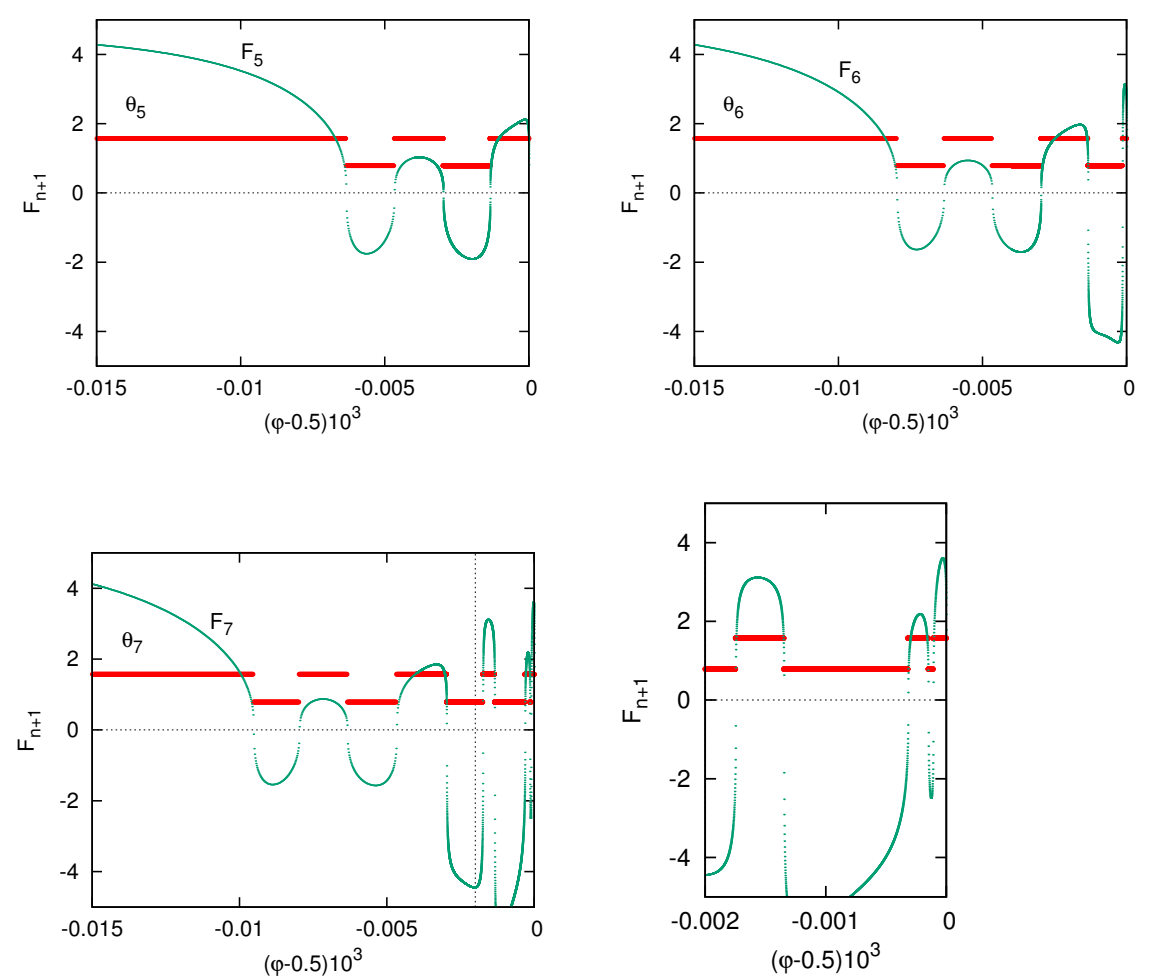

Figure 7: Functions $\theta_{n+1}(\varphi)$ and $F_{n+1}(\varphi)=r\left(\theta-\theta_{c}\right)$ for $\alpha=1, h=-1, \varphi<1 / 2$ and $n=4,5$ (first row) and $n=6$ (second row; on the right, the plot shows a detail showing five of the zeros of the function). $\alpha=1, h=-1$. 
We want to notice two important issues. On one hand, the bigger the order $n$, the smaller the interval $I=(1 / 2-\varepsilon, 1 / 2+\varepsilon)$ where some of the zeros of $F_{n+1}$ exist. However, fixed an order $n$, to look for the zeros of $F_{n+1}$ with a good accuracy, it is important to take a big $\varepsilon$, and a suitable $\xi$ (as big as possible). This implies that a high order approximation of the parametrization of the invariant manifold is needed.

On the other hand, up to the values of $n$ computed, we find that all of the zeros of the functions $F_{n+1}$ are transversal. If this observation was true for any ECO, the result of the Proposition 5 would be an if and only if result. That is, if the ECO of order $n$ corresponds to $\bar{\varphi}$ and $\Gamma$ is the solution with initial condition $\Psi(\bar{\varphi})$, such that

$$
P(\Gamma)=\left(\bar{p}_{1}, \ldots, \bar{p}_{n}\right)
$$

then

$$
\begin{aligned}
& P_{n+1}(\bar{\varphi}+\epsilon)=\left(\bar{p}_{1}, \ldots, \bar{p}_{n}, p_{n+1}^{1}\right) \\
& P_{n+1}(\bar{\varphi}-\epsilon)=\left(\bar{p}_{1}, \ldots, \bar{p}_{n}, p_{n+1}^{2}\right)
\end{aligned}
$$

${ }_{435}$ where $p_{n+1}^{1} \neq p_{n+1}^{2}$ for $\epsilon$ small enough. Numerically we observe that this is true.

\subsection{Results}

We present here, for $\alpha=1$ and $h=-1$, the ECO computed up to order $n \leq 7$ by looking for the zeros of the function $F_{8}(\varphi)$ as explained in the previous Section. The orbits obtained are summarized in Tables 1- 4. Recall that, for any ECO of order $n$ of type $\left(p_{1}, \ldots, p_{n}\right)$, there exists also the symmetric one $\left(p_{n}, \ldots, p_{1}\right)$ that traces the same path in configuration space, so they are not included.

For $n \leq 4$, only ECO of type $(1, ., n, 1)$ or $(2, . ., n, 2)$ exist, so there are only two ECOs for each order, see Table 1 For $n=5$ we find four different ECO, all of them symmetric, see Table 2 The first non-symmetric orbits are found for $n \geq 6$. For $n=6$, there exist eight different ECOs, two symmetric and six non-symmetric, see Table 3. We plot three of the non-symmetric ones, the 
(1)

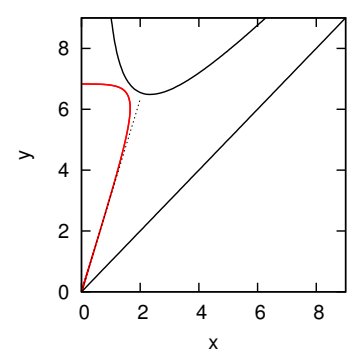

$(2,2)$

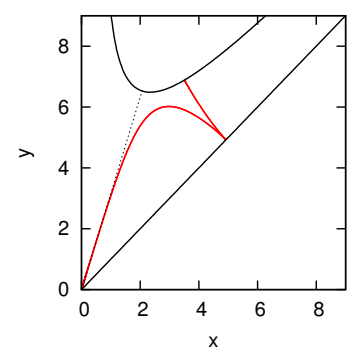

$(1,1,1,1)$



$(2)$

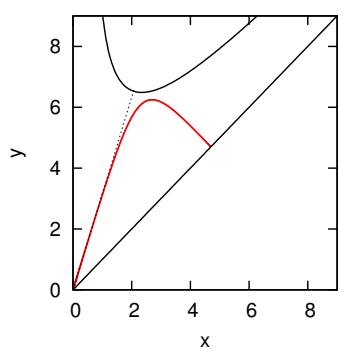

$(1,1,1)$

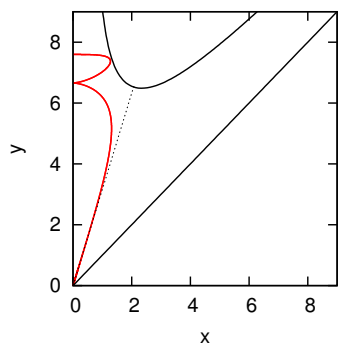

$(2,2,2,2)$

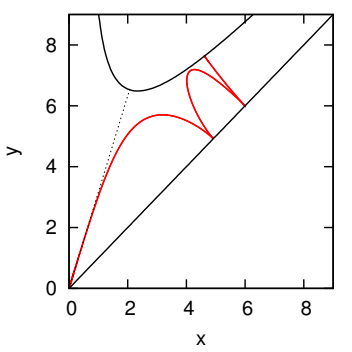

$(1,1)$

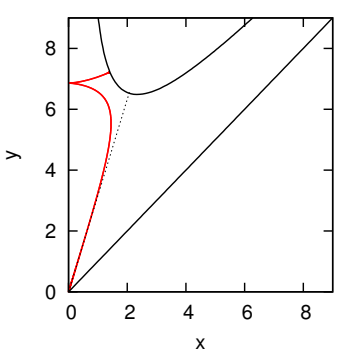

$(2,2,2)$

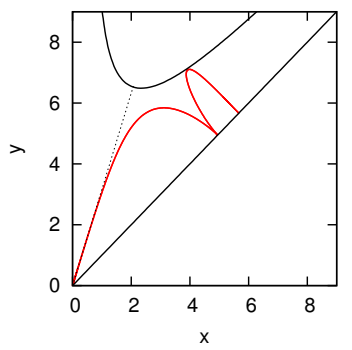

Table 1: For each order $n=1, \ldots, 4$ only two ECOs are found, all of them exhibiting binary collisions of only one type for $\alpha=1$ and $h=-1$. 
other three are obtained by symmetry, and have the same projection in the configuration space. Similarly, for $n=7$, there exists twelve different ECOs, four of them symmetric, see Table 4.

$(1, ., ., 1)$

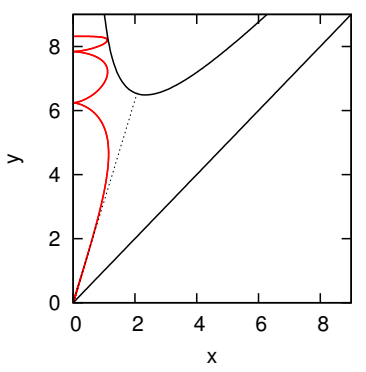

$(1,2,1,2,1)$

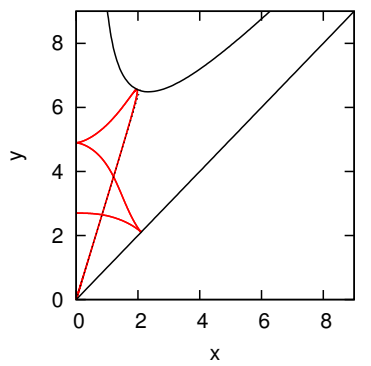

$(2, ., ., 2)$

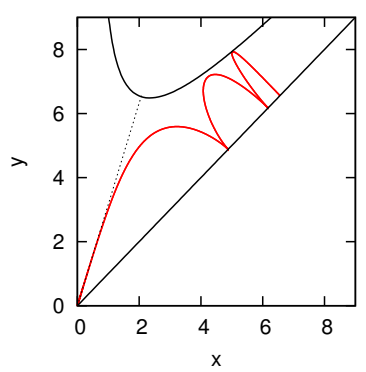

$(2,1,2,1,2)$



Table 2: ECOs obtained for $n=5$ with $\alpha=1$ and $h=-1$. All of them are symmetric.

Notice that, as was stated in Proposition 4, when $n$ is even, the symmetric orbits have a point on the zero velocity curve.

We finally notice that in 20, Lacomba and Medina give a graph, for certain values of $\alpha$, that allows to identify the possible sequences of binary collisions for an orbit passing near the total collision. This is in accordance with [12, where the authors mention that not all the possible sequences are realizable. We reproduce (in our notation) that graph in Figure 8 for $\alpha=1$. For example, from the graph in Figure 8 it is clear, that no ECO orbits of types $(2, k), 2,1,1)$ and $(1, \ldots) ., 1,2,2)$ can exist (since from the graph, we see that the partial sequences ${ }_{460}(2,1,1)$ or $(1,2,2)$ cannot exist). 


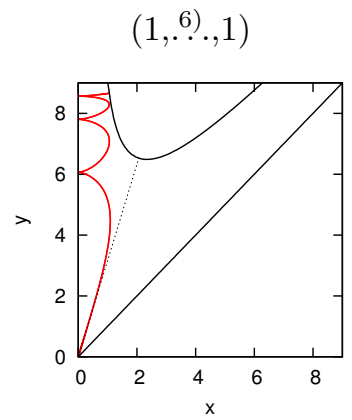

$(2,6) ., 2)$

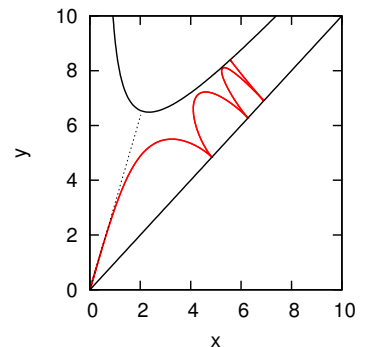

$(1,2,1,2,1,2)$

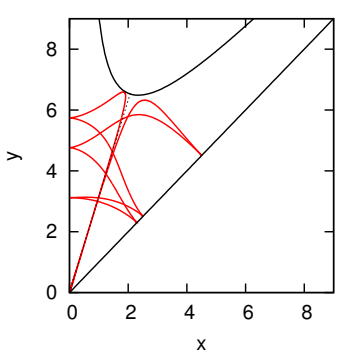

$(1,1,2,1,2,1)$

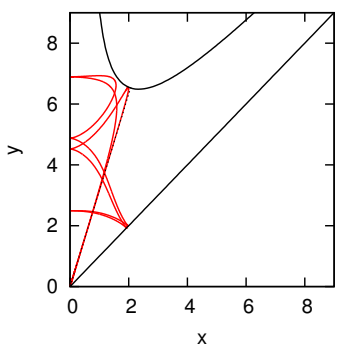

$(2,2,1,2,1,2)$

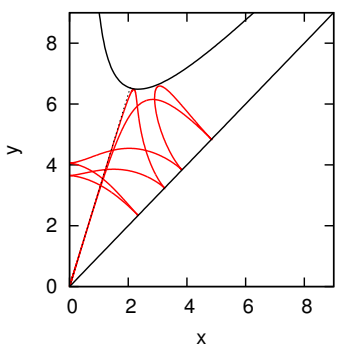

Table 3: ECOs obtained for $n=6$ with $\alpha=1$ and $h=-1$. First row, symmetric orbits. Second row, non-symmetric orbits. Their symmetric ones, which have the same projection on the $(x, y)$ plane, are the ECOs $(2,1,2,1,2,1),(1,2,1,2,1,1)$ and $(2,1,2,1,2,2)$. 
$(1,7) ., 1)$

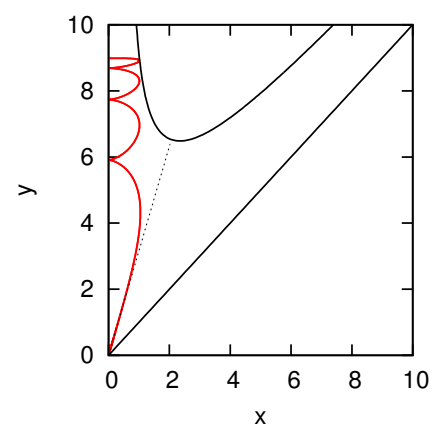

$(1,1,2,1,2,1,1)$

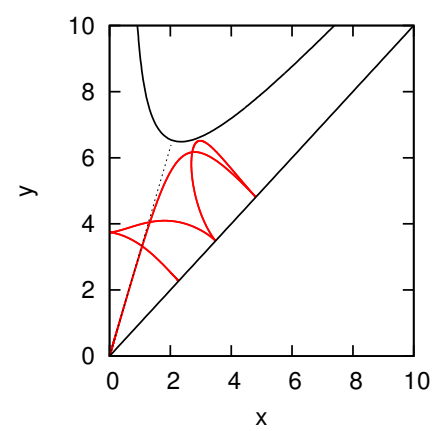

$(1,1,1,2,1,2,1)$

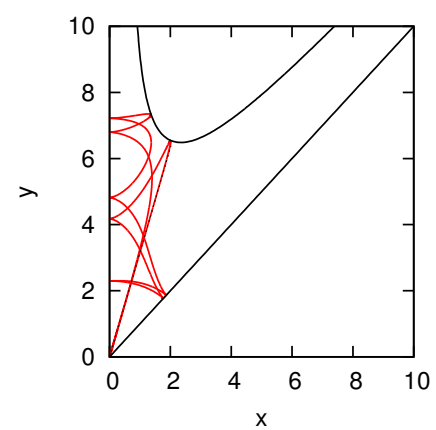

$(1,2,1,2,1,2,2)$

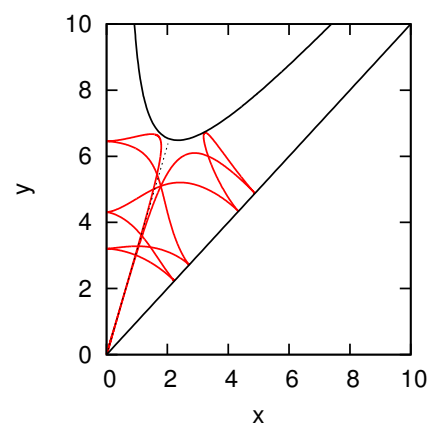

$(2,7) ., 2)$

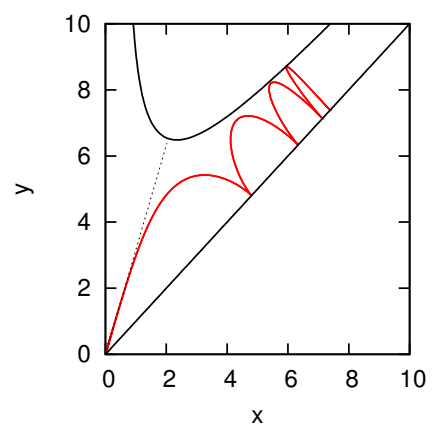

$(2,2,1,2,1,2,2)$

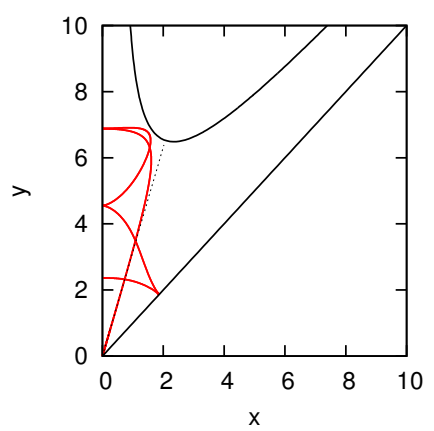

$(1,1,2,1,2,1,2)$

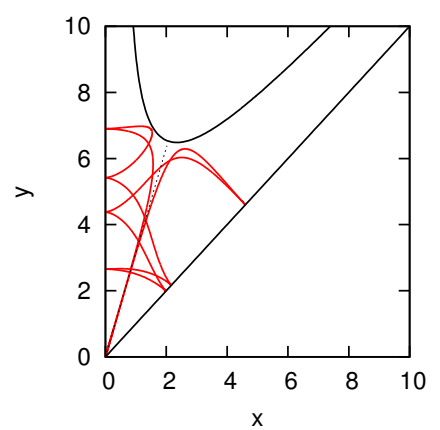

$(2,2,2,1,2,1,2)$

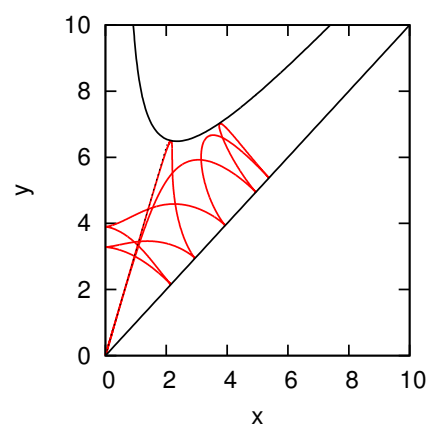

30

Table 4: ECOs for $n=7$ with $\alpha=1$ and $h=-1$. Symmetric ones (first and second rows). Non symmetric ones (third and fourth rows). Their symmetric ones, which have the same projection on the $(x, y)$ plane, are the ECOs $(1,2,1,2,1,1,1),(2,1,2,1,2,1,1),(2,2,1,2,1,2,1)$ and $(2,1,2,1,2,2,2)$. 


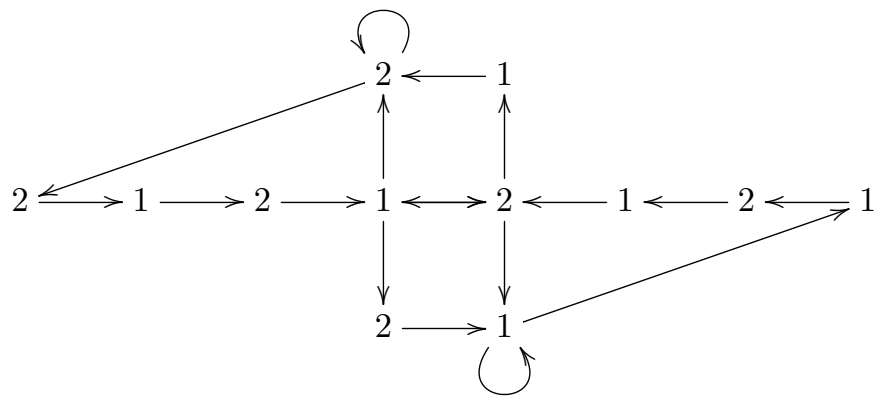

Figure 8: Directed graph containing allowed sequences of binary collisions for orbits passing near the total collision for $\alpha=1$.

\section{Discussion and conclusions}

As stated in 12, the invariant manifolds $W^{s, u}\left(E^{ \pm}\right)$separate different kinds of dynamical behaviors taking into account the type of binary collisions. In particular, their intersections correspond to the ejection-collision orbits from/to quadruple collision. On one hand, we present here a specific characterization of the ECO and a methodology to compute them for any value of the mass parameter $\alpha$ and negative energy $h$. The method presented uses as a Poincaré section $\Sigma_{c}$ that corresponds to the binary collisions DBC and SBC. The difference with the mentioned work is that they use as a Poincaré section the plane that contain the homothetic $\left\{\theta=\theta_{c}\right\}$, which is not suitable for the computation of the ECOs. The methodology also relies in the computation of an approximation of order $m$ (for a suitable $m$ ) of the parametrization of the invariant manifolds. On the other hand, the approximated parametrization allows us to prove that there exist orbits that eject from quadruple collision and escape to 475 infinity performing only binary collisions of one type (direct escape). Clearly, the same is true reversing time (orbits ending in quadruple collision). Our guess is that there should be a separation between those ejection orbits that escape directly with one type of binary collisions and the remaining ejection-escape orbits (with several mixed types of binary collisions). Of course, this separation should be visible by computing the connections between the invariant manifolds of $E^{+}$and $E^{-}$and the manifolds of the infinity. But this is work for a future paper. 
Although the methodology described applies for any value of $\alpha>0, h<0$ and any given order $n$ for the ECO, we present the results obtained for $\alpha=1$ and $h=-1$, and show ECO up to order $n=7$. We also remark that different results, concerning the type of ECO, may be expected depending on $\alpha$. More precisely, for $\alpha=1$, we notice that if there exists an ECO of type $\left(p_{1}, \ldots, p_{n}\right)$, then we also find the ECO of type $\left(\widehat{p}_{1}, \ldots, \widehat{p}_{n}\right)$, with $\widehat{p}_{j}=3-p_{j}$. This seems to be related with the same topological behaviour, concerning SBC and DBC, of the invariant manifolds of $E^{ \pm}$on the quadruple collision manifold (but no analytical proof is known so far). However, as shown in Figure 3 for $\alpha=2$, the behaviour of such manifolds, and therefore the type of ECO obtained, varies with $\alpha$.

\section{Appendix}

Here we present in detail some results concerning the eigenvalues and eigenvectors associated to the equilibrium points for any values of $\alpha$ and $h<0$. We give their expressions in terms of $h, v_{c}$ and $\theta_{c}$, and the explicit formulae for the approximations of the invariant manifolds of order one $\Psi_{1}^{ \pm}(\xi, \phi)$.

Recall that the equilibrium points are $E^{ \pm}=\left(0, \pm v_{c}, \theta_{c}, 0\right)$, where $\theta_{c}$ is the minimum of $V$ (defined in (4)) and $v_{c}^{2}=V\left(\theta_{c}\right)$. Then, $V^{\prime}\left(\theta_{c}\right)=0$ and $V^{\prime \prime}\left(\theta_{c}\right)>$ 0 . The eigenvalues associated to the equilibrium points are

$$
\begin{array}{ll}
\lambda_{1}\left(E^{ \pm}\right)= \pm \lambda, & \lambda_{2}\left(E^{ \pm}\right)=-\lambda_{1}, \\
\lambda_{3}\left(E^{ \pm}\right)=\frac{\lambda}{4}\left(\mp 1+\sqrt{1+\frac{8 V^{\prime \prime}\left(\theta_{c}\right)}{V\left(\theta_{c}\right)}}\right), & \lambda_{4}\left(E^{ \pm}\right)=\frac{\lambda}{4}\left(\mp 1-\sqrt{1+\frac{8 V^{\prime \prime}\left(\theta_{c}\right)}{V\left(\theta_{c}\right)}}\right),
\end{array}
$$

where $\lambda=\sqrt{2 \cos \theta_{c}\left(\sin \theta_{c}-\sqrt{\alpha} \cos \theta_{c}\right)}>0$. Notice that $\lambda_{3}>0$ and $\lambda_{4}<0$, and $\lambda_{3} \neq \lambda_{4}$. Next we prove that all the eigenvalues are different for any value of $\alpha$.

Lemma 1. Let $\lambda_{i}\left(E^{ \pm}\right), i=1, \ldots, 4$, be the eigenvalues associated to the equilibrium points $E^{ \pm}$of the $S C 4 B P$. Then, for all values of $\alpha$ :

1. all the eigenvalues are different: $\lambda_{i} \neq \lambda_{j}, i \neq j$, 
Proof. Notice that, from [18], the two inequalities of the second statement are equivalent, so it is enough to prove one of them. Moreover, it is not difficult to see that both statements are equivalent to

$$
V^{\prime \prime}\left(\theta_{c}\right)-3 V\left(\theta_{c}\right)>0 \quad \text { and } \quad V^{\prime \prime}\left(\theta_{c}\right)-V\left(\theta_{c}\right) \neq 0
$$

where $\theta_{c}$ is the unique solution of $V^{\prime}(\theta)=0$, and $V$ is given in (4). We write $V(\theta)=\frac{\sqrt{2}}{2 \cos \theta} h(z)$ where $z=\tan \theta>\sqrt{\alpha}$ and

$$
h(z)=1+\frac{\alpha^{5 / 2}}{z}+8 \alpha^{3 / 2} \frac{z}{z^{2}-\alpha} .
$$

Then

$$
\begin{aligned}
V^{\prime}(\theta)= & \frac{\sqrt{2}}{2 \cos \theta}\left(z h(z)+h^{\prime}(z)\left(z^{2}+1\right)\right), \\
V^{\prime \prime}(\theta)= & \frac{\sqrt{2}}{2 \cos \theta}\left(z\left(z h(z)+h^{\prime}(z)\left(z^{2}+1\right)\right)\right. \\
& \left.+\left(h(z)+3 z h^{\prime}(z)+\left(z^{2}+1\right) h^{\prime \prime}(z)\right)\left(z^{2}+1\right)\right),
\end{aligned}
$$

where the prime' denotes derivative with respect $z$.

The condition $V^{\prime}\left(\theta_{c}\right)=0$ is equivalent to

$$
z_{c} h\left(z_{c}\right)+h^{\prime}\left(z_{c}\right)\left(z_{c}^{2}+1\right)=0
$$

where $z_{c}=\tan \left(\theta_{c}\right)$. Introducing this relation into the expression for $V^{\prime \prime}\left(\theta_{c}\right)$, we have that the conditions in 19 become

$$
V^{\prime \prime}\left(\theta_{c}\right)-3 V\left(\theta_{c}\right)=\frac{\sqrt{2}}{2 \cos \theta_{c}}\left(1+z_{c}^{2}\right)\left(\left(1+z_{c}^{2}\right) h^{\prime \prime}\left(z_{c}\right)-2 h\left(z_{c}\right)\right)>0
$$

and

$$
V^{\prime \prime}\left(\theta_{c}\right)-V\left(\theta_{c}\right)=\frac{\sqrt{2}}{2 \cos \theta_{c}}\left(\left(1+z_{c}^{2}\right)^{2} h^{\prime \prime}\left(z_{c}\right)-2 z_{c}^{2} h\left(z_{c}\right)\right) \neq 0,
$$

where $z_{c}$ is the solution of 20 .

We introduce the change $z_{c}=\sqrt{\alpha} w$ in 20 , where $w>1$. Simplifying, the equation transforms into

$$
w^{7}-2 w^{5}-(8+17 \alpha) w^{4}+w^{3}+(2 \alpha-8) w^{2}-\alpha=0
$$


The equation is linear in $\alpha$, and $17 w^{4}-2 w^{2}+1>0$ for $w>1$. Therefore, we can write

$$
\alpha=\frac{w^{2}\left(w^{5}-2 w^{3}-8 w^{2}+w-8\right)}{17 w^{4}-2 w^{2}+1} .
$$

Imposing the condition $\alpha>0$, we have that $w>\bar{w}$, where $\bar{w} \in[11 / 5,12 / 5]$ is the only positive root of $w^{5}-2 w^{3}-8 w^{2}+w-8=0$.

We introduce the change $z_{c}=\sqrt{\alpha} w$ in 21 and 22 , and simplifying and keeping the numerators, we get that these conditions are equivalent, respectively, to

$$
w^{9}-3 w^{7}-(41 \alpha+8) w^{6}+3 w^{5}+(11 \alpha-24) w^{4}-w^{3}-3 \alpha w^{2}+\alpha>0
$$

and

$$
\begin{aligned}
\alpha w^{11}-3 \alpha w^{9}-\left(42 \alpha^{2}+16 \alpha\right) w^{8}+3 \alpha w^{7}+\left(14 \alpha^{2}-49 \alpha-8\right) w^{6} \\
-\alpha w^{5}-\left(6 \alpha^{2}-3 \alpha+24\right) w^{4}+\left(2 \alpha^{2}-3 \alpha\right) w^{2}+\alpha \neq 0 .
\end{aligned}
$$

Finally, we introduce 23 in the above expressions, and they are equivalent to

$$
\begin{aligned}
& (w-1)(w+1)\left(3 w^{2}+1\right)\left(w^{7}-w^{5}-8 w^{4}+w^{2}-1\right)>0 \\
& (w-1)^{5}(w+1)^{3}\left(w^{7}-w^{5}-8 w^{4}+w^{2}-1\right) \\
& \quad \times\left(25 w^{5}+50 w^{4}+80 w^{3}+46 w^{2}+15 w+8\right) \neq 0
\end{aligned}
$$

for $w>\bar{w}$. The computation of the zeros of the term $w^{7}-w^{5}-8 w^{4}+w^{2}-1$ gives that it is positive for $w>11 / 5$, so both statements are true.

The first result of Lemma 1 implies that for all values of $\alpha$ there exist 2dimensional invariant manifolds $W^{u}\left(E^{+}\right)$and $W^{s}\left(E^{-}\right)$. The tangent space to the unstable manifold $W^{u}\left(E^{+}\right)$is generated by the eigenvectors associated to $\lambda_{1}$ and $\lambda_{3}$. The tangent space to the stable manifold $W^{s}\left(E^{-}\right)$is generated by the eigenvectors associated to $\lambda_{1}$ and $\lambda_{4}$. The corresponding eigenvectors $\sigma_{i}\left(E^{ \pm}\right)$ 
can also be written in terms of $\theta_{c}, v_{c}$ and the energy as

$$
\begin{aligned}
\sigma_{1}\left(E^{ \pm}\right) & =\left(-v_{c}, \mp h, 0,0\right), \\
\sigma_{3}\left(E^{ \pm}\right) & =\left(0,0,1, \lambda_{3}\right), \\
\sigma_{4}\left(E^{ \pm}\right) & =\left(0,0,1, \lambda_{4}\right) .
\end{aligned}
$$

Therefore, we can write the approximations of order one of the parametrizations of the invariant manifolds. For example, using the normalized eigenvectors $\bar{\sigma}_{i}=\sigma_{i} /\left\|\sigma_{i}\right\|$, we have that for $W^{u}\left(E^{+}\right)$:

$$
\begin{aligned}
\Psi_{1}^{+}(\xi, \phi) & =E^{+}+\xi\left(\cos (2 \pi \varphi) \bar{\sigma}_{1}+\sin (2 \pi \varphi) \bar{\sigma}_{3}\right) \\
& =\left(\begin{array}{c}
0 \\
v_{c} \\
\theta_{c} \\
0
\end{array}\right)+\xi\left(\begin{array}{c}
\frac{-v_{c}}{\sqrt{h^{2}+v_{c}^{2}}} \cos (2 \pi \varphi) \\
\frac{-h}{\sqrt{h^{2}+v_{c}^{2}}} \cos (2 \pi \varphi) \\
\frac{1}{\sqrt{1+\lambda_{3}^{2}}} \sin (2 \pi \varphi) \\
\frac{\lambda_{3}}{\sqrt{1+\lambda_{3}^{2}}} \sin (2 \pi \varphi)
\end{array}\right) .
\end{aligned}
$$

From the second statement of Lemma 1 we have that the eigenvector associated to $\lambda_{1}$ gives the slow direction in both invariant manifolds, whereas the strong directions are given by the eigenvectors associated to $\lambda_{3}$ (for $W^{u}\left(E^{+}\right)$) 525 and $\lambda_{4}$ (for $\left.W^{s}\left(E^{-}\right)\right)$.

As we noticed in Section 2.2 in order to have $r>0$, we need $\cos (2 \pi \varphi)<0$, so we consider $\varphi \in(1 / 4,3 / 4)$. The values $\varphi=1 / 4,3 / 4$ give the solutions along the direction $\sigma_{3}$ or $\sigma_{4}$ (the fast direction). In this case, $r=0$ and we obtain orbits inside the collision manifold. The value $\varphi=1 / 2$ gives the solution along the direction $\sigma_{1}$ (the slow direction), which corresponds to the homothetic solution. Notice that we could have taken $\sigma_{1}\left(E^{ \pm}\right)=\left(v_{c}, \pm h, 0,0\right)$. In this case, we must consider $\varphi \in(-1 / 4,1 / 4)$, and the fast and slow directions would correspond to $\varphi= \pm 1 / 4$ and $\varphi=0$, respectively. 


\section{Acknowledgements}

535

UE), and Catalan (AGAUR) grant 2017 SGR 1374. M. Ollé is partially supported by Spanish MNECO/FEDER grant MTM2015-65715 and the Catalan grant 2017SGR1049.

\section{References}

[7] P. Leonard, Stellar collisions in globular clusters and the blue straggler problem, Astron. J. 98 (1989) 217-226.

[8] J. Fregeau, P. Cheung, S. Portegies Zwart, F. Rasio, Stellar collisions during binary-binary and binary-single star interactions, Mon. Not. R. Astron. Soc. 352 (2004) 1-19. 
[9] M. Álvarez-Ramírez, M. Medina, A model for binary-binary close encounters and collisions from a dynamical point of view, Astrophys. Space Sci. 349 (2014) 143-150.

[10] R. McGehee, Triple collision in the collinear three-body problem, Invent. Math. 27 (1974) 191-227. URL https://doi .org/10.1007/BF01390175

[11] W. Sweatman, The symmetrical one-dimensional Newtonian four-body problem: a numerical investigation, Celest. Mech. Dyn. Astron. 82 (2) (2002) 179-201, the restless universe (Blair Atholl, 2000).

URL https://doi.org/10.1023/A:1014599918133

[12] M. Sekiguchi, K. Tanikawa, On the symmetric collinear four-body problem, Publ. Astron. Soc. Jpn. 56 (2004) 235-251.

[13] L. Bakker, T. Ouyang, S. Simmons, D. Yan, G. Roberts, Linear stability for some symmetric periodic simultaneous binary collision orbits in the four-body problem, Celest. Mech. Dyn. Astron. 108 (2010) 147-164.

575 [14] W. Sweatman, A family of symmetrical Schubart-like interplay orbits and their stability in the one-dimensional four-body problem, Celest. Mech. Dyn. Astron. 94 (1) (2006) 37-65.

URL https://doi.org/10.1007/s10569-005-2289-8

[15] T. Ouyang, D. Yan, Periodic solutions with alternating singularities in the collinear four-body problem, Celest. Mech. Dyn. Astron. 109 (2011) 229239.

[16] H.-Y. Huang, Schubart-like orbits in the newtonian collinear four-body problem, DCDS, A 32 (2012) 1763-1774.

[17] M. Álvarez-Ramírez, M. Medina, C. Vidal, The trapezoidal collinear fourbody problem., Astrophys. Space Sci. 358 (2015) 1-17. 
[18] J. Mather, R. McGehee, Dynamical Systems, Theory and Applications, Vol. 38 of Lecture Notes in Physics, Springer, Berlin, Heidelberg, 1975, Ch. Solutions of the collinear four body problem which become unbounded in finite time., pp. 573-597.

[19] A. Haro, M. Canadell, J. Figueras, A. Luque, J. Mondelo, The parameterization method for invariant manifolds. From Rigorous Results to Effective Computations., Vol. 195 of Applied Mathematical Science, Springer-Verlag, 2016.

[20] E. Lacomba, M. Medina, Symbolic dynamics in the symmetric collinear four-body problem, Qual. Theory Dyn. Syst. 5 (1) (2004) 75-100.

URL https://doi.org/10.1007/BF02968131

[21] D. Saari, Collisions, rings, and other Newtonian $N$-body problems, Vol. 104 of CBMS Regional Conference Series in Mathematics, Published for the Conference Board of the Mathematical Sciences, Washington, DC; by the American Mathematical Society, Providence, RI, 2005.

URL https://doi.org/10.1090/cbms/104

[22] E. Lacomba, Infinity manifolds for positive energy in celestial mechanics, in: The Lefschetz centennial conference, Part III (Mexico City, 1984), Vol. 58 of Contemp. Math., Amer. Math. Soc., Providence, RI, 1987, pp. 193-201. 